\title{
Innovations in Molecular Identification of Mycobacterium tuberculosis
}

\author{
Mohammad Irfan ${ }^{1}$ iD and Dakshina Bisht ${ }^{2 *}$ (D) \\ ${ }^{1}$ Department of Clinical Laboratory Science, College of Applied Medical Science-Ad Dawadmi, \\ Shaqra University, 17464 Ad Dawadmi, Kingdom of Saudi Arabia. \\ ${ }^{2}$ Department of Microbiology, Santosh Medical College, Santosh University, Ghaziabad - 201009 , \\ Uttar Pradesh, India.
}

\begin{abstract}
Tuberculosis (TB) continues to be a significant public health concern on a global scale. Quick and precise identification of Mycobacterium tuberculosis (MTB) in symptomatic patients is pivotal for worldwide TB eradication initiatives. As an infectious disorder induced by MTB, it remains a critical threat to public health, particularly in poor countries, due to an inadequate diagnostic research laboratory. There is a need for a persistent incentive to reduce response time for effective diagnosis and control of TB infection, which is a benefit that molecular techniques provide over traditional methods. Although there is a tremendous overall prevalence of TB and a relatively poor probability of case identification worldwide. Common screening techniques have focused on tests that have many fundamental shortcomings. Due to the development of antibiotic-resistant Mycobacterium strains, TB is one of the leading contributors to fatalities. It is now possible to examine TB using molecular detection techniques, which are faster and more cost-effective than previous methods, such as standard culture procedures to test and verify antibiotic resistance in patients with TB. Whole genome sequencing (WGS), faster nucleic acid amplification tests, has made it easier to diagnose and treat TB more quickly. This article addresses the genetic approaches for detecting Mycobacterium tuberculosis complex (MTBC) in clinical specimens as well as antibiotic resistance in mycobacterium and discusses the practical limitations of using these methods.
\end{abstract}

Keywords: Nucleic acid amplification test, tuberculosis, Mycobacterium tuberculosis complex (MTBC), diagnosis of tuberculosis, Molecular Identification

*Correspondence: dakshinabisht@gmail.com; +91 9810510852

(Received: December 14, 2021; accepted: February 2, 2022)

Citation: Irfan M, Bisht D. Innovations in Molecular Identification of Mycobacterium tuberculosis. J Pure Appl Microbiol. 2022;16(1):74-88. https://doi.org/10.22207/JPAM.16.1.76

(C) The Author(s) 2022. Open Access. This article is distributed under the terms of the Creative Commons Attribution 4.0 International License which permits unrestricted use, sharing, distribution, and reproduction in any medium, provided you give appropriate credit to the original author(s) and the source, provide a link to the Creative Commons license, and indicate if changes were made. 


\section{INTRODUCTION}

In 2019, approximately 10 million people across the world were infected with TB. TB affects people of all ages and in all nations. Fortunately, it is possible to treat and prevent TB if caught early. ${ }^{1}$ India is leading the way with two-thirds of the total cases. ${ }^{1}$ As reported by WHO in 2020, TB is one of the top ten diseases that cause mortality and the most common cause of death caused by a single infectious pathogen, placing above HIV/ AIDS. During the COVID-19 pandemic, there was an increase of approximately $13 \%$, bringing a total of approximately $1.66(1.3-2.1)$ million TB deaths in $2020 .^{2}$

Multidrug-resistant TB (MDR-TB) continues to be an issue and a possible threat to public health. In 2019, a net of 206030 people with MDR-TB have been identified and reported worldwide, that is, a $10 \%$ increase from 186883 in 2018. ${ }^{1}$ Globally, the rate of TB infection is falling to $9 \%$ between 2015 and 2019. ${ }^{1}$ This is less than halfway to meeting the End TB Strategy goal of $20 \%$ reduction between 2015 and 2020. ${ }^{1}$ In the year between 2019 and 2020, approximately 60 million individuals were spared via diagnosis and treatment approaches. The United Nations Sustainable Development Goals (SDGs) include a health objective of eradicating TB by 2030.

MDR-TB and Extensively drug-resistant TB (XDR-TB), two forms of drug-resistant, are on the rise in several regions of the globe. The advent of drug-resistant TB highlights the inadequacies of TB eradication efforts and the healthcare system's failure to prevent resistant forms of illness from emerging. Despite significant advances in TB detection, less than half of all pulmonary $T B$ cases globally were laboratory verified. TB control can be improved by developing and using more sensitive and effective diagnostic technologies that can diagnose MTB quickly and accurately. ${ }^{3}$ The most widely used method for diagnosing TB is microscopy and it is ineffective in HIVpositive people and children. ${ }^{4}$ Furthermore, the outcome of this screening does not distinguish between Mycobacterium species. ${ }^{5}$ Alternatively, Lowenstein-Jensen culture, which is often considered the gold standard in presumptive respiratory infections, looks to be more specific than smear microscopy, however, it is more timeconsuming (4-8 weeks in culture). It demands appropriate infrastructure and possibly the best laboratory personnel ${ }^{6}$ which can cause delays in efficient medical treatments. Therefore, the demand for novel, quick, and reliable diagnostic procedures has arisen.

Because the traditional bacteriological assessment of TB has numerous drawbacks, the NAAT (Nucleic Acid Amplification Test) has emerged as a viable substitute. ${ }^{7}$ NAAT systems have fast turnaround periods, uncomplicated testing and because of that treatment may begin on the same visit. Also, cutting the cost and frequency of follow-ups. ${ }^{8}$ For the detection of MTBC organisms, most NAAT tests look for mycobacterial insertion element. ${ }^{9}$ NAAT identifies MTB ribosomal RNA or DNA in sputum samples. ${ }^{10}$ NAAT has very high precision in smear-positive patients and a sensitivity range of $61-76 \%$ in smear-negative individuals. ${ }^{11}$ With the tremendous expansion of molecular methods, a broad range of NAAT to determine the presence of TB are now accessible. Polymerase chain reaction (PCR), real-time $P C R$, and loop-mediated isothermal amplification (LAMP) are examples of such techniques.

Rapid drug susceptibility tests (DST) and molecular diagnostic tests for MTBC identification would perhaps be an explanation for our prevailing TB diagnostic inadequacies. These diagnostics, unlike growth-based assays, depend on genetic information and are completed in hours instead of weeks. These assays have revolutionized the TB diagnostic paradigm enabling quick and precise diagnosis in TB-endemic locations wherever the culture-based assessment was formerly inaccessible. This review addresses the genetic approaches for detecting MTBC in clinical specimens as well as antibiotic resistance in mycobacterium and discusses the practical limitations of using these methods. Our search criteria included tuberculosis, drug resistance, early diagnosis, and molecular diagnostic approaches. We used PubMed, Scopus, and Google to find relevant articles. In this study, only peer-reviewed articles were considered.

Historically Significant Milestones in Tuberculosis Analysis

The growth of novel molecular approaches for the diagnosis of TB has accelerated in recent years. Nevertheless, that wasn't always 
the scenario before the PCR was introduced in 1985. The following are examples of the earliest commercially available PCR-based approaches for detecting MTBC.

The Mycobacterium tuberculosis direct test (MTD)

The FDA authorized the MTD test, an isothermal method, in December 1995, to detect MTBC rRNA using smear-positive respiratory samples. The MTD test uses transcription-mediated amplification (TMA) and the hybridization protection assay (HPA2) to qualitatively identify MTBC rRNA. Following CDC recommendations, the Grady Memorial Hospital microbiology laboratory introduced the amplified Mycobacterium tuberculosis Direct Test (MTD), a kind of NAAT from Hologic Gen-Probe (San Diego, CA) in $2000^{11}$. According to the manufacturer. ${ }^{12}$ It was shown to have great sensitivity (96.9\%) and specificity (100\%) when used for smear positive specimens. This suggests that it could be beneficial not only for the rapid detection of TB but also helpful in differentiating between TB and nontuberculous mycobacteria (NTM) strains.

\section{Enhanced amplified Mycobacterium tuberculosis direct test (E-AMTDT)}

The FDA has authorized the E-AMTDT (Gen-Probe ${ }^{\circledR}$, Inc., San Diego, CA) for the immediate evaluation of MTBC in respiratory samples obtained from individuals suspected of having TB. ${ }^{13}$ It is an RNA-based approach that depends on Kwoh's isothermal transcription-mediated amplification mechanism. ${ }^{14}$ The significant aspects of this test are the speed and the reduced threat of cross-contamination. The foremost drawback of E-AMTDT is its failure to identify inhibitors because it lacks an internal amplification control (IAC).

\section{COBAS Amplicor MTB Test}

This test was approved in 1996. The Roche Diagnostic Systems Cobas Amplicor PCR system for MTB detection (CA-PCR; Branchburg, NJ, USA) integrates specimen processing with controlled proliferation and diagnosis. The Cobas Amplicor test relies on biotinylated primers, a capture probe, and photometric labelling ${ }^{15}$ to quantify a 584-bp 5' segment of the $16 \mathrm{~S}$ rRNA gene $^{16}$. This technique was thoroughly tested for diverse clinical samples and exhibited good sensitivity and specificity, principally for smearpositive samples. ${ }^{17}$
Ligase chain reaction (LCX) MTB Assay

LCX MTB (Abbott Diagnostics Division, Abbott Park, III) intercepts MTBC in respiratory specimens using the ligase chain reaction. LCR depends on the combination of two particular oligonucleotide probes for adjoining sequences in the target DNA, a stretch of the MTB chromosome that specifies the protein antigen $b$, which is processed sequentially by polymerase and ligase. This gene sequence is unique to the MTBC. An enzyme conjugate trapped by the amplified ligase product enables the substrate to generate a fluorescence signal proportional to its concentration in the LCx analyzer. The cut-off determines whether the findings are positive or negative. During the last several years, multiple reports ${ }^{18}$ evaluated the sensitivity, specificity, and clinical usefulness of the LCX-MTB test for speedy identification of $M T B C$ in respiratory and non-respiratory samples. They demonstrated that the LCX-MTB test is an effective TB diagnostic method. ${ }^{19}$ The Abbott LCx Probe System is quite costly due to the limited resources of the microbiological laboratory in underdeveloped countries, where TB has always been a major public health concern. Therefore, the LCx-MTB test can be housed in a single central laboratory that collaborates with local microbiological laboratories and clinical facilities. As a result, this test must be evaluated in situations that include specimen storage and transit from various locations. In 2002, this assay was pulled from the European market. ${ }^{20}$ BD ProbeTec-SDA (strand displacement amplification)

Becton Dickinson (Sparks, MD) designed a semiautomated technique designated BD Probe Tec for the quick recognition of MTBC in respiratory specimens. ${ }^{21}$ The underlying principle used was a thermophilic variant of strand displacement amplification (SDA) that exponentially duplicated target nucleic acid sequences in measurable quantities. ${ }^{22}$ Becton Dickinson has successfully developed the latest system called BD ProbeTec ET, a novel device that connects SDA to a fluorescent energy transfer (thus, the "ET" designation) sensing mechanism. The BD ProbeTec ET system at the same time replicates and recognizes samples in a sealed homogeneous assay format, ${ }^{23}$ leading to increased productivity as well as a much speedier assessment. 


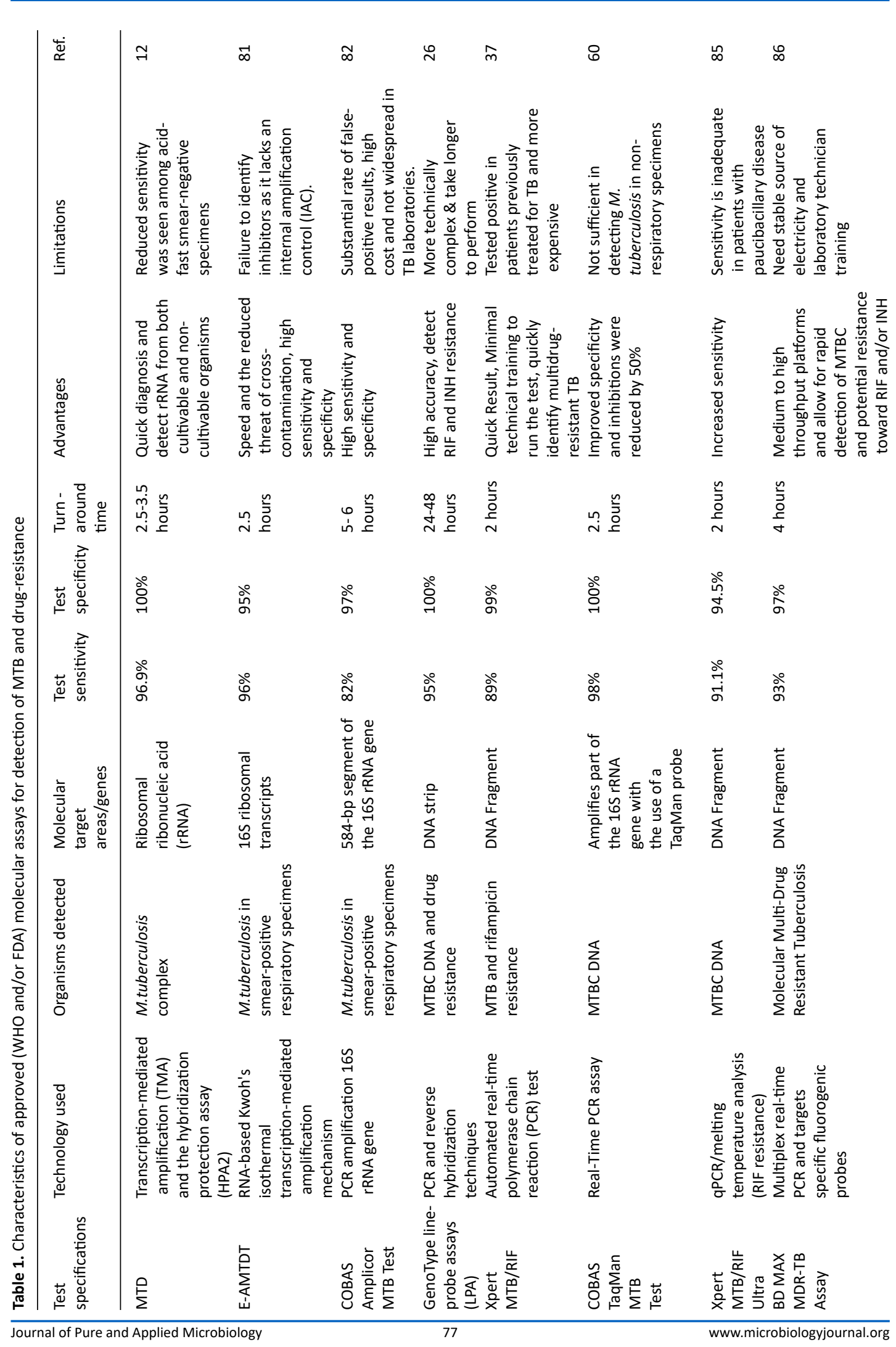




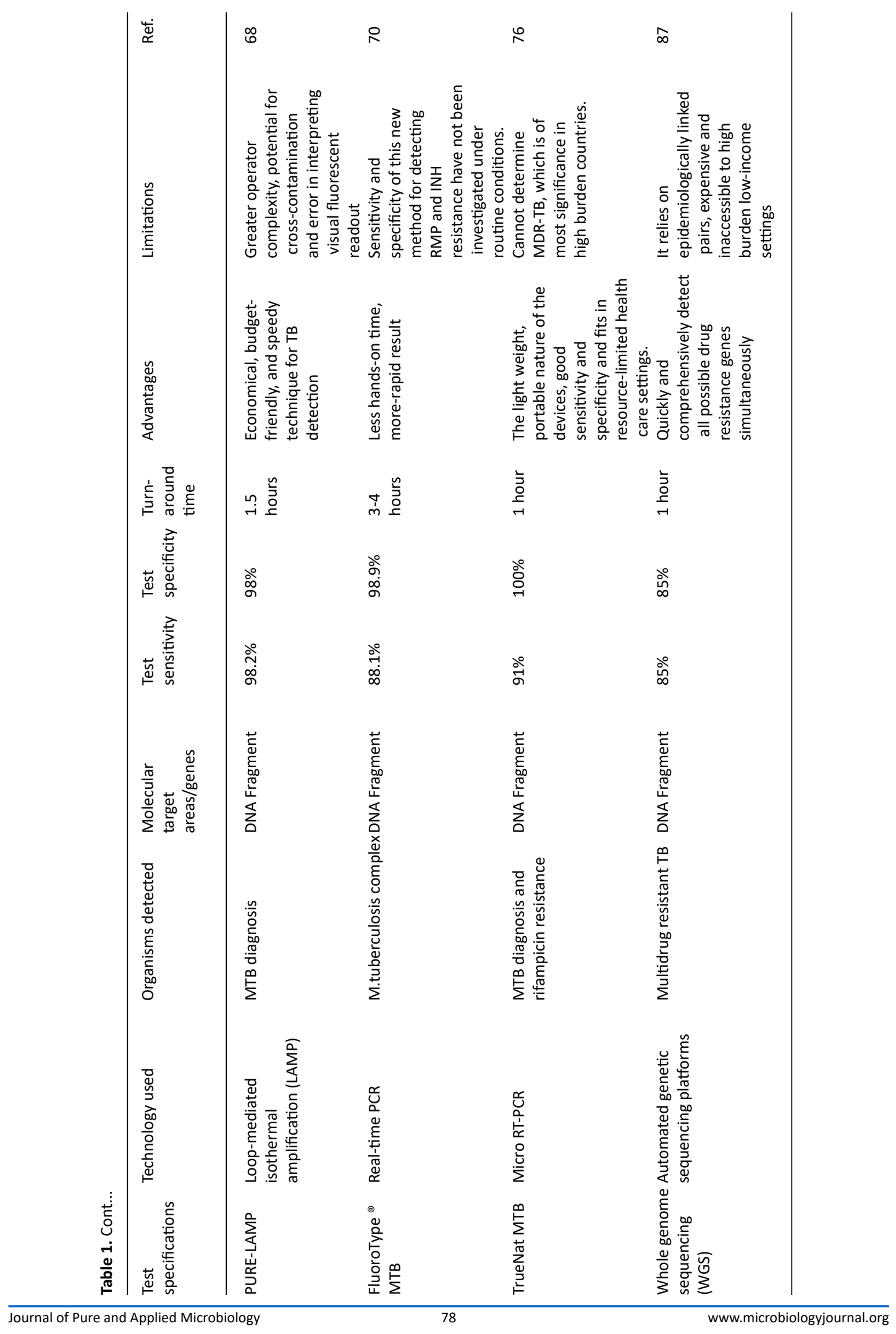


In the era of antibiotic resistance, none of these techniques proved to be easy to use and cheap enough to be truly adequate for TB laboratory routine diagnosis. ${ }^{24}$

Innovations In Techniques for Molecular Diagnostics of Tuberculosis

Molecular detection of MTBC has advanced significantly with the discovery of genetic variants related to resistance to the most widely used first- and second-line TB drugs. These techniques employ an extensive range of targets, including genus or species-specific DNA or RNA segments.

\section{GenoType line-probe assays (LPA)}

Molecular tests for screening gene alterations that indicate drug resistance are considered ideal for prompt diagnosis, particularly because assays can be used directly on diagnostic samples. ${ }^{25}$ LPAs have demonstrated considerable potential among molecular assays. ${ }^{26}$ LPA is a class of newer DNA strip-based assays that employ NAAT like PCR and reverse hybridization techniques to detect drug resistance mutations rapidly. Currently, there are two accessible LPAs, that is, the INNO-LiPA ${ }^{\circledR}$ Rif TB assay and the GenoType ${ }^{\circledR}$ MTBDRplus assay. Hain Lifescience, a German company, invented Genotype MTBDRplus. INNO-LiPA Rif. TB was developed by Innogenetics, a Belgian company.

Innogenetics introduced the INNOLiPA Rif.TB assay in 1995, the first commercially accessible and widely used line probe assay generally accessible for detecting MTBC DNA and drug resistance mutations. ${ }^{27}$ LiPA managed to give results within one to two days. It was approved by the WHO in 2008. A meta-analysis by the WHO looked at the performance of the LiPA assay. It found that it had a sensitivity higher than $95 \%$ and a specificity of $100 \%$ for the identification of MTBC isolates. ${ }^{26}$ When the LiPA was used directly on clinical samples, it had $100 \%$ specificity and sensitivity that varied from $80 \%$ to $100 \% .{ }^{26}$

Hain Lifescience introduced the GenoType MTBDR, a qualitative in-vitro test in 2004. The Genotype MTBDR assay targets rpoB RPDR and kat $G$ codon 315 which are possibly the most common. The research compared to phenotypic DST, MTBDR has a sensitivity and specificity of $99 \%$ and $100 \%$ for rifampicin resistance, respectively, and $88.4 \%$ and $100 \%$ for isoniazid resistance, respectively. ${ }^{28}$

The limited sensitivity of the test to detect isoniazid resistance was its main drawback in achieving a significant improvement in the number of isoniazid-resistant strains. In 2007, Hain made a second-generation test called Genotype MTBDRplus VER 1.0 (MTBDRplus). This is for all genes that the previous version targeted, but adds codons 505 to 510 to the RRDR of the rpoB gene. In 2008, the WHO validated MTBDRplus VER $1.0^{26}$. The genotype MTBDRplus VER 2.0 was approved by WHO in 2012 as a quantifiable in-vitro methodology for the identification of the MTBC and instantaneous sensing of mutations in the rpoB and katG genes. This test takes around 6 hours to complete. ${ }^{29}$ Investigations ${ }^{30-31}$ indicate that the analytical effectiveness of Genotype MTBDRplus Version 2.0 LPA for the identification of MDR-TB in direct smear-positive sputum samples was remarkably accurate and explicit. Furthermore, the reliability of the assay to detect MDR-TB in direct smear-negative sputum samples must be strengthened..$^{32}$ All the chemicals essential for amplification in this assay are included in the amplification mix and they are no longer required to be administered separately. Processing time is comparable. MTBDRplus exhibited a high rate of recognition of resistance to rifampicin in both MTB strains and clinical specimens.

The GenoType MTBDRsl test is the third in the sequence of LPA assays. The test uses the same technology as its predecessor but recognizes mutations synonymous with second-line drug resistance. Specifically, to improve the capability to identify low-level kanamycin tolerance, MTBDRsI VER 2.0 was developed in 2015 to enhance the efficiency of the GenoType MTBDRsl assay. The GenoType MTBDRsl 2.0-line probe assay ${ }^{33}$ was devised as a means to identify MTB, as well as to simultaneously recognize the presence of resistance-inducing mutations in fluoroquinolones (gyrA and gyrB genes) and second-line intravenous medications (SLID) (rrs and eis genes). ${ }^{34}$ The particular section for the screening of resistance to ethambutol (embB codon 306) that was included in MTBDRsl v1 has been deleted from MTBDRsI v2.0. WHO advised using the GenoType MTBDRsI 2.0 assay rather than phenotypic DST. ${ }^{35}$ 


\section{Gene Xpert TB Assays/ Xpert MTB/RIF}

The Xpert MTB/RIF assay is a novel technique that redefines TB management and facilitates rapid detection of TB disease. Xpert MTB/RIF (Cepheid Inc.) is a GeneXpert-based automated real-time PCR assay (Fig. 1). ${ }^{36}$ The time it takes to identify MTB and rifampicin resistance after starting the analysis is just around two hours with little technical effort. ${ }^{37}$ Both the WHO and the US Food and Drug Administration (FDA) have given their approval for its use. ${ }^{38-40}$ This technique may be employed on clinical specimens including pure sputum samples as well as samples generated after disinfection and concentration of sputum..$^{41}$ When compared to previous approaches, many studies ${ }^{42-43}$ have shown that Xpert MTB/RIF is a more precise approach for quick TB screening. Upon a negative response to AFB smear microscopy, Xpert MTB/RIF can be utilized as a first as well as a supplementary assay to confirm the diagnosis ${ }^{37}$ and has minimized the current estimate for therapy for AFB smearnegative TB from 56 to 5 days. ${ }^{44}$ Xpert MTB/RIF, the leading primary diagnostic test for $\mathrm{TB}$ and antibiotic resistance, is an in-vitro screening test introduced in 2009 to be used within the fully automated GeneXpert platform..$^{45}$ In 2013, FDA approved the Xpert MTB/RIF. ${ }^{46}$ A systematic assessment of the specificity and accuracy of the Xpert MTB/RIF for TB screening predicted pooled diagnostic accuracy of $89 \%$ - $99 \%$ correspondingly. ${ }^{37}$ In culture-positive patients with negative smear, the pooled sensibility was $67 \%$. Xpert MTB/RIF assay's important clinical significance is that it is fully automated, safe, and quickly recognizes MTB DNA and genetic alterations that cause rifampicin resistance as a biomarker for MDR-TB. Several investigations revealed significant limits in the performance of the Xpert MTB/RIF test. Among them is the inability to identify rifampicin resistance-related alterations in mixtures ${ }^{47}$ and the inability to identify the $\mathrm{C} 533 \mathrm{G} \mathrm{rpoB}$ mutation that is correlated with rifampicin susceptibility. ${ }^{48}$ A revised variant of the test, the Xpert MTB/RIF Ultra, has been created to increase the sensitivity and accuracy of the assay.

Loop-mediated isothermal amplification-based MTB detection assay (LAMP)

Notomi et al.,49 from Eiken Chemical Company- Japan, designed the LAMP test. LAMP has been effectively used as a diagnostic method in clinical research. ${ }^{50}$ LAMP is an isothermal nucleic acid multiplication technology that utilizes a constant temperature to amplify nucleic acids without the necessity of a thermal cycler. Under isothermal circumstances, this technique

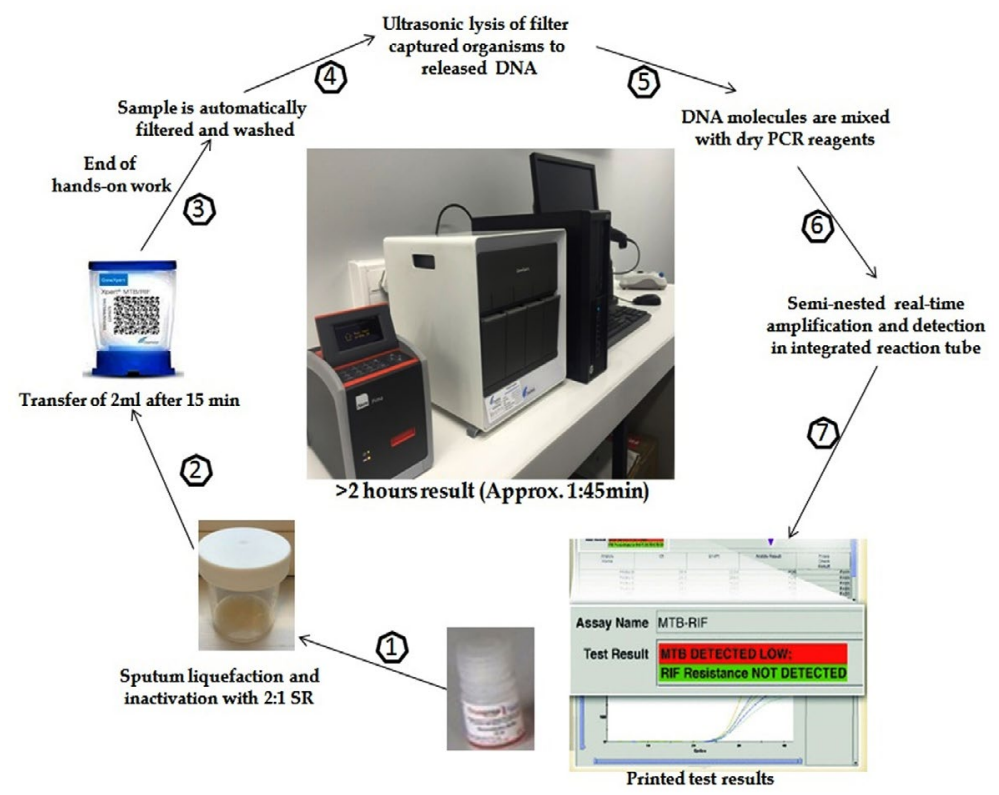

Fig. 1. Xpert MTB/RIF Assay Overview. 


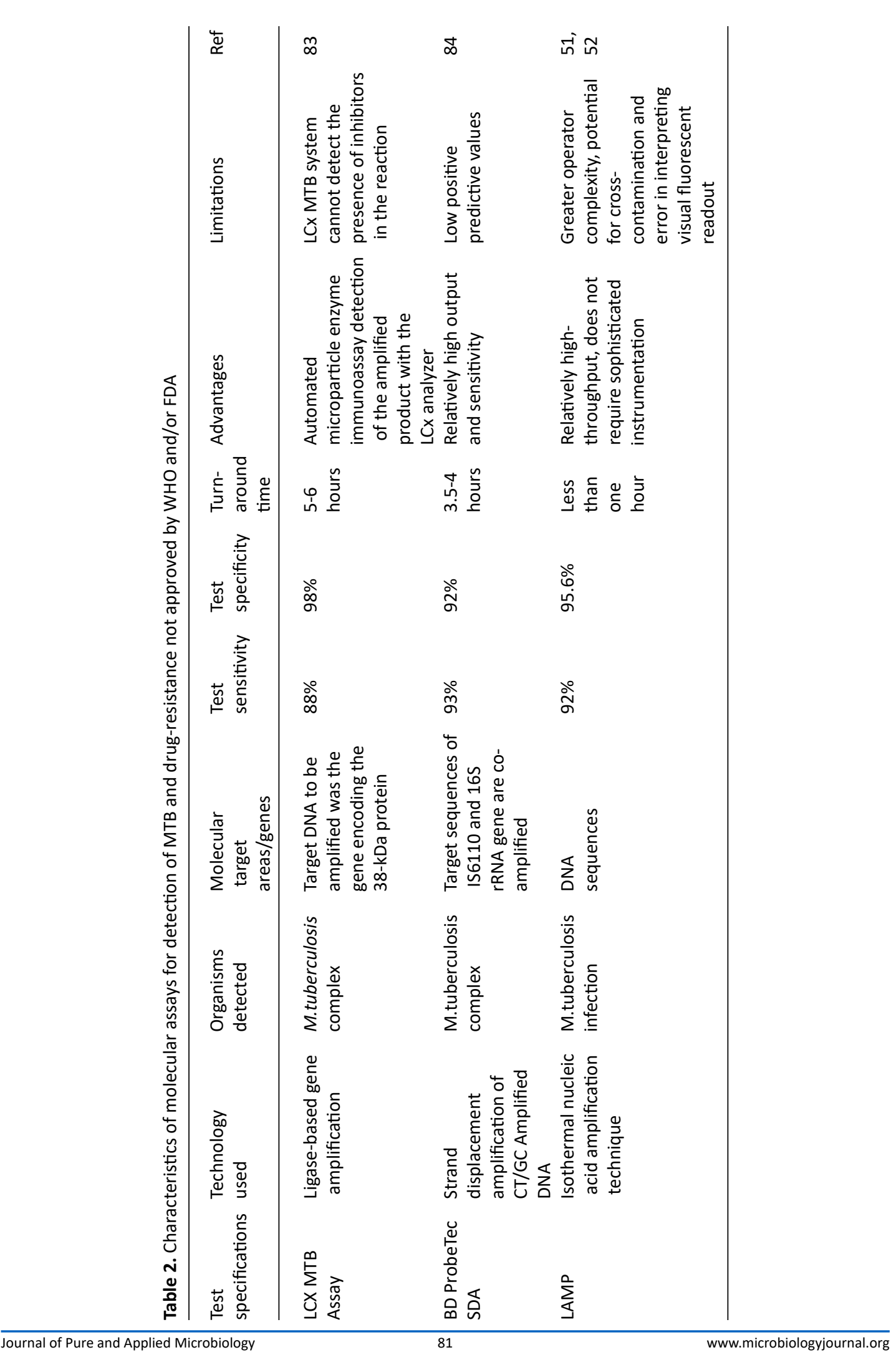


multiplies relatively few copies of DNA sequences with excellent accuracy, reliability, and efficiency employing a combination of four specially engineered primers and a DNA polymerase with strand displacement capability. ${ }^{49}$ LAMP was proposed by WHO in August 2016 as a viable substitute for smear microscopy for evaluating pulmonary TB in individuals. For smear-positive specimens, TB-LAMP had a stronger specificity of $92.1 \%-100 \%$ compared to smear-negative samples $52.1 \%-90.3 \% .^{51}$ A recent investigation demonstrates that LAMP showed a high sensitivity (95.6\%) in extra-pulmonary TB samples when contrasted to traditional methods. ${ }^{52}$ The efficacy of TB-LAMP assessment using extrapulmonary samples is currently being investigated. ${ }^{53}$ The TBLAMP can turn out to be the ultimate molecular test for TB detection; however, excessive heat, moisture, insufficient reagent amount, and crosscontamination amongst specimens have been highlighted as key contributors to false-positive responses. ${ }^{54}$

\section{Promising Developments in TB Molecular Diagnostics}

Based on the current WHO flow path for the TB molecular assessment, innovative advancements are currently in different phases of progression with the vast majority still in the initial phases of development while others are present in the WHO appraisal and are portrayed below.

\section{COBAS TaqMan MTB (CTM Test)}

A new MTB test, the Cobas TaqMan MTB test, has been introduced by Roche Diagnostics (Rotkreuz, Switzerland), which has superseded the Cobas Amplicor MTB test. This technique is based on real-time PCR that uses a TaqMan probe to intensify the $16 \mathrm{~S}$ rRNA gene segment to recognize MTBC DNA in clinical samples. ${ }^{57}$ According to the manufacturer's recommendations, CTM should only be used on respiratory samples. ${ }^{55}$ However, several investigations have been conducted to assess the efficacy of the CTM test in nonrespiratory specimens. ${ }^{56-57}$ Studies indicate that the Cobas test is more accurate regarding smearpositive samples than it is regarding smearnegative samples ${ }^{58}$ that could be associated with the purification and concentration stages. ${ }^{59}$ The assay's sensitivity varied from $96.9 \%-98 \%$ in the smear-positive sample as well as $34.9 \%-79.5 \%$ in the smear-negative samples. Additionally, the specificity ranges from $78.1 \%-100 \%$ in smearpositive samples as well as from $98.7 \%-99 \%$ in smear-negative samples. ${ }^{60}$ However, these findings differ from one research to another. According to some studies, this variation is attributable due to the AFB smear condition, distinctive specimen categories, and the prevalence of TB. ${ }^{61}$ Thus, the Cobas TaqMan MTB test findings should indeed be thoroughly evaluated in association with the clinical evidence.

\section{Xpert MTB/RIF Ultra}

The Xpert MTB/RIF Ultra has a larger reaction area and also has two genetic markers, IS6110 and IS1081 in it. All of this works together to lower the detection limit to $15.6 \mathrm{CFU} / \mathrm{ml}^{62}$ Also, it increases the sensitivity up to ten times ${ }^{62}$. Xpert Ultra also performed better in the following areas: identification of susceptibility in unknown compounds, identification of alterations at codon 533, and differentiating silent alterations at codons 513 and $514 .^{62}$ The WHO recommends this as early diagnostic tool for symptomatic TB patients. ${ }^{63}$

\section{GeneXpert Omni and Xpert XDR}

Cepheid intends to introduce the GeneXpert Omni, a significant technical development for evaluating TB and rifampicin resistance that will use the same cartridges as the present in GeneXpert equipment. The GeneXpert Omni is a single-cartridge portable diagnostics machine that cost less than other GeneXpert tests and can run for four hours on a single charge. Due to the aforementioned qualities, it is extremely helpful, particularly in rural areas with inadequate infrastructure, for quick identification of TB. Omni was scheduled to be released in developing markets at the end of 2018; however, the WHO has not yet marketed or reviewed it. ${ }^{64}$ The Xpert $^{\circledR}$ MTB/XDR cartridge enables clinicians to quickly prescribe treatment regimens for MDR-TB and XDR-TB. ${ }^{65-66}$

\section{BD MAX MDR-TB Assay}

It was developed by Becton and Dickinson to detect MTBC DNA and mutations that give resistance to rifampicin and isoniazid. It is a fully automated machine that utilizes multiplex real-time PCR and targets specific fluorogenic probes. ${ }^{69}$ Target the mutation in the rpoB and katG genes and in the inhA promoter region. This assay was specifically designed for analyzing raw or concentrated sputum. The BD MAX MDRTB 
test was introduced in June 2018. The research demonstrated good sensitivity and specificity. ${ }^{67}$ PURE-LAMP

The PURE-LAMP is a quantitative TB diagnostic assay designed by Eiken Chemical Company-Japan between 2007-2010. This test could be performed in approximately 90 minutes and consists of the following steps, i.e., sample processing, LAMP amplification, and visual evaluation of fluorescent illumination from the reaction tube using ultraviolet rays. ${ }^{68}$ Several studies have shown that PURE-LAMP has very significant diagnostic accuracy, making it an economical, budget-friendly, and speedy technique for TB detection. ${ }^{69}$ According to research, ${ }^{68}$ the sensitivity of the test is $55.6 \%$ in patients with a negative smear and $98.2 \%$ in patients with a positive smear. According to N'guessan et al., ${ }^{6}$ the PURE-LAMP test has a greater sensitivity than that of smear microscopy. However, the specificity of the smear microscopy was greater than that of PURE-LAMP. ${ }^{6}$ Thus, PURE-LAMP should be used in conjunction with many other analytical procedures to support the presence of TB, particularly in falsenegative samples. ${ }^{70}$

\section{FluoroType ${ }^{\circledR}$ MTB}

FluoroType ${ }^{\circledR}$ MTB analysis (Hain Lifescience, Nehren, Germany) detects MTBC in pulmonary and non-pulmonary clinical isolates using real-time PCR. The HyBeacon fluorescence technique $^{71}$ has been used in the FluoroType ${ }^{\circledR}$ MTB assessment. The amplifications were performed using Hain Lifescience FluoroCycler equipment, and the analysis was performed with a melt response curve with single-stranded DNA fragments marked with fluorescent dyes that are complementary to the amplified DNA. The technique yields responses in 3-4 hours for numerous samples. ${ }^{72}$ In contrast to culture, Hofmann-Thiel and Hoffmann ${ }^{72}$ discovered that the FluoroType ${ }^{\circledR}$ MTB test exhibited a sensitivity of $88.1 \%$ and a specificity of $98.9 \%$. There are two versions of FluoroType ${ }^{\circledR}$ MTB. FluoroType MTB VER 1.0 is used for decontaminated pulmonary and extrapulmonary samples, and FluoroType ${ }^{\circledR}$ MTB VER 2.0 is used for decontaminated pulmonary samples. $^{73}$

\section{The TrueNat MTB}

It is a novel approach that can identify resistance to MTB and rifampicin in one hour. The TrueNat model was implemented by the Indian company MolBio Diagnostics Pvt Ltd, Goa. The Bigtec Labs in India financed its development. As the complete system is battery-powered and mobile, it may be employed at the most elementary tiers of the healthcare system. It functions by efficiently screening for TB bacteria using the PCR assay. The isolated DNA is then put onto a TrueNat MTB (or MTB plus) chip that has already been preloaded with stabilized reagents.

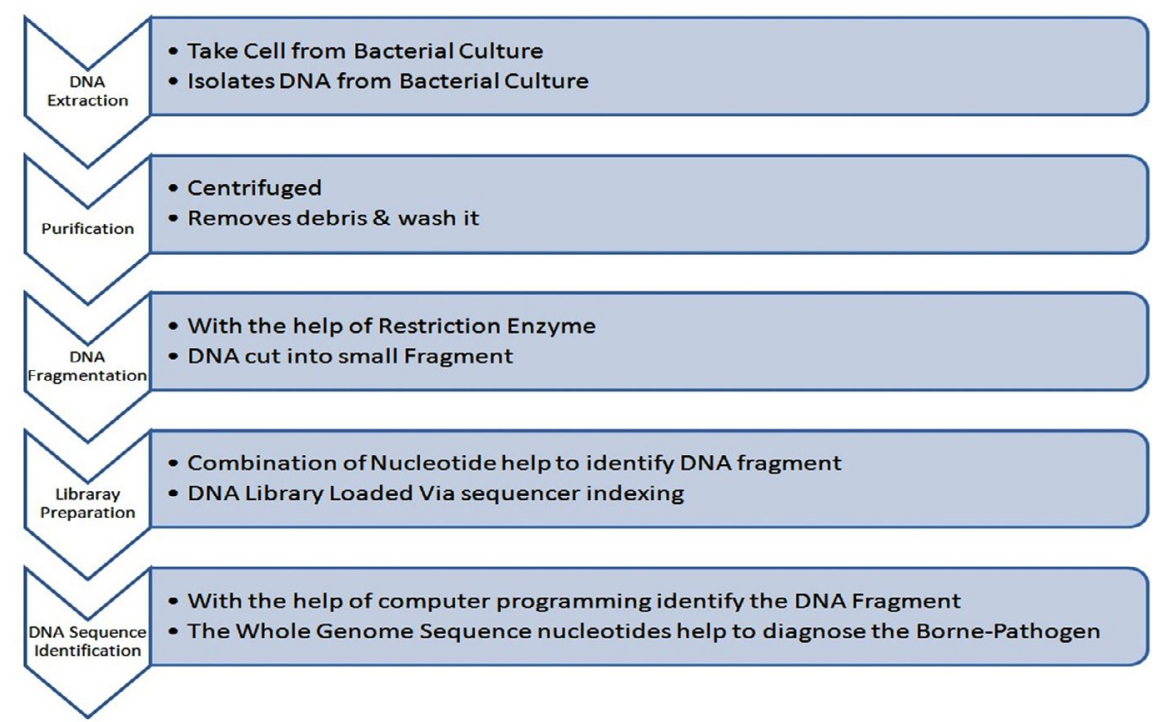

Fig. 2. Procedure of Whole-Genome Sequencing from Specimen Preparation to Diagnostic Evaluation 
The Truelab UnoDx real-time PCR analyzer, a portable battery-powered instrument, is then used for rapid amplification and analysis. If the TrueNat MTB chip (or MTB plus) produces a positive result (depending on the diagnostic algorithm used), the user can then insert another aliquot of extracted DNA into an additional TrueNat MTB-RIF Dx chip to identify the presence of specific mutations linked to resistance to rifampicin. ${ }^{74}$ The system searches for DNA that is unique to the TB bacterium. If it is detected, the machine will utilize PCR to amplify specific segments of DNA, which may subsequently be employed in a variety of laboratory processes. If resistance to rifampicin is discovered, a second RT-PCR was performed. ${ }^{75}$ The TrueNat MTB test demonstrated $91 \%$ sensitivity and $100 \%$ specificity against a composite reference standard in a pilot study conducted in India, including $99 \%$ specificity for smear-positive, culture-positive samples and $76 \%$ sensitivity for smear-negative, culturepositive samples. ${ }^{76}$

\section{Whole-genome sequencing (WGS)}

WGS techniques, which use automated genetic sequencing platforms, have been suggested as the most effective molecular diagnostic test for MTB and the identification of all genetic factors for resistance. ${ }^{77}$ Microbial biotech has made it possible to look at the genetic markers of organisms that could affect treatment and infection prediction. ${ }^{78}$

WGS has now become an economical \& easy approach to identify microevolutions within MTB lineages when they are transferred across hosts. ${ }^{79}$ The WGS workflow is depicted in Fig. 2, showing sample collection to clinical diagnostic evaluation. First-generation and secondgeneration sequencers (commonly known as the next-generation sequencer) are available. Despite its slowness, the first-generation sequencer has a maximum speed and a low price. The second generation seems to have lesser productivity and higher expense and can sequence numerous genomes in much less than a day. ${ }^{80}$

\section{CONCLUSIONS}

The future of molecular diagnostic analysis for active TB of the lungs appears promising and it should be developed in a way that improves public health. Molecular methodologies certainly had a significant influence on patient care and have been one of the most significant methods in recent years to validate the presence of MTB in the laboratory. Even while this approach is useful in mycobacteriology labs, it isn't the only way to address all questions. We must be aware of the limits of these methods when they are used. These incorporate technical glitches such as suppression of amplification, cross-contamination, and significantly lower accessibility; the need for expensive reagents, and input costs; constrained multiplexing potential; significantly longer response times, particularly for DNA retrieval, and the need for technical knowledge and specific amenities. To determine whether a particular diagnostic molecular technology is suitable for a specific clinical environment, factors such as testing capacity, diagnostic yield, and practicality of performing the test in the laboratory must be taken into account.

\section{ACKNOWLEDGMENTS}

None.

\section{CONFLICT OF INTEREST}

The authors declare that there is no conflict of interest.

\section{AUTHORS' CONTRIBUTION}

All authors listed have made a substantial, direct and intellectual contribution to the work, and approved it for publication.

\section{FUNDING}

None.

\section{ETHICS STATEMENTS}

Not Applicable.

\section{DATA AVAILABILITY}

All datasets generated or analyzed during this study are included in the manuscript.

\section{REFERENCES}

1. Global tuberculosis report 2019. Accessed October 14, 2020. https://www.who.int/publications/i/ item/9789241565714

2. Glaziou P. Predicted impact of the COVID-19 pandemic on global tuberculosis deaths in 2020, Global TB Programme, World Health Organization, Switzerland. 2020. doi: 10.1101/2020.04.28.20079582v1

3. Dicks KV, Stout JE. Molecular Diagnostics for Mycobacterium tuberculosis Infection. Annu Rev Med. 2019;70:77-90. doi: 10.1146/annurev- 
med-040717-051502

4. Dunn JJ, Starke JR, Revell PA. Laboratory diagnosis of Mycobacterium tuberculosis infection and disease in children. J Clin Microbiol. 2016;54(6):1434-1441. doi: 10.1128/JCM.03043-15

5. Dezemon Z, Muvunyi CM, Jacob O. Staining techniques for detection of acid fast bacilli: what hope does fluorescein-diacetate (FDA) vitality staining technique represent for the monitoring of tuberculosis treatment in resource limited settings. Trends Bacteriol. 2014;1(1):1-6. doi: 10.7243/2057-4711-1-1

6. N'guessan K, Adegbele J, Coulibaly I, et al. Clinical performances of Pure TB-Lamp kit for $M$. tuberculosis complex detection in sputum samples. J Tuberc Res. 2017;5(2):129-138. doi: 10.4236/jtr.2017.52014

7. Qin L, Zheng R, Fan C, et al. Identification and evaluation of a new nucleic acid amplification test target for specific detection of Mycobacterium tuberculosis. Clin Chem Lab Med. 2010;48(10):15011505. doi: 10.1515/CCLM.2010.296

8. Niemz A, Boyle DS. Nucleic acid testing for tuberculosis at the point-of-care in high-burden countries. Expert Rev Mol Diagn. 2012;12(7):687-701. doi: 10.1586/ erm.12.71

9. Tiwari S, Nataraj G, Kanade S, Mehta P. Diagnosis of pediatric pulmonary tuberculosis with special reference to polymerase chain reaction based nucleic acid amplification test. Int J Mycobacteriol. 2015;4(1):48-53. doi: 10.1016/j.jimyco.2014.11.063

10. Sia IG, Wieland ML. Current concepts in the management of tuberculosis. Mayo Clin Proc. 2011;86(4):348-361. doi: $10.4065 / \mathrm{mcp} .2010 .0820$

11. Centers for Disease Control and Prevention. Updated guidelines for the use of nucleic acid amplification tests in the diagnosis of tuberculosis. MMWR Morb Mortal Wkly Rep. 2009;58(1):7-10. https://pubmed.ncbi.nlm. nih.gov/19145221/

12. Amplified MTD Test | Hologic. Accessed June 1, 2014. http://www.hologic.ca/products/clinical-diagnosticsblood-screening/assays-and-tests/amplified-mtd-test

13. Centers for Disease Control and Prevention. Update: nucleic acid amplification tests for tuberculosis. MMWR Morb Mortal Wkly Rep. 2000;49(26):593-594. https://pubmed.ncbi.nlm.nih.gov/10921499/

14. Kwoh DY, Davis GR, Whitfield KM, Chappelle HL, DiMichele LJ, Gingeras TR. Transcription-based amplification system and detection of amplified human immunodeficiency virus type 1 with a bead-based sandwich hybridization format. Proc Natl Acad Sci. 1989;86(4):1173-1177. doi: 10.1073/pnas.86.4.1173

15. Kirschner P, Rosenau J, Springer B, Teschner K, Feldmann K, Bottger EC. Diagnosis of mycobacterial infections by nucleic acid amplification: 18-month prospective study. J Clin Microbiol. 1996;34(2):304 -312. doi: 10.1128/jcm.34.2.304-312.1996

16. Roche Diagnostics. Cobas Amplicor Mycobacterium tuberculosis test: instruction manual. Roche Diagnostics, 2007; Mannheim, Germany. https:// scholar.google.com/scholar_lookup?title=Cobas $\% 20$ Amplicor\%20Mycobacterium\%20tuberculosis\%20 test\%3A\%20instruction\%20manual\&publication_ year $=2007$
17. Piersimoni C, Scarparo C. Relevance of commercial amplification methods for direct detection of Mycobacterium tuberculosis complex in clinical samples. J Clin Microbiol. 2003;41(12):5355-5365. doi: 10.1128/JCM.41.12.5355-5365.2003

18. Viinanen AH, Liippo K, Soini $H$, Marjamaki M, Viljanen MK. Ligase chain reaction assay is clinically useful in the discrimination of smearpositive pulmonary tuberculosis from atypical mycobacterioses. Ann Med. 2000;32(4):279-283. doi: 10.3109/07853890009011773

19. Viveiros M, Pinheiro S, Moreira P, Pacheco T, Brum L. Evaluation of a commercial ligase chain reaction assay for the diagnosis of pulmonary and extra-pulmonary tuberculosis. Int J Tuberc Lung Dis. 1999;3(6):508-514. https://pubmed.ncbi.nlm.nih.gov/10383064/

20. Kwiatkowska S, Marczak J, Zieba M, Nowak D. Clinical utility of a commercial ligase chain reaction kit for the diagnosis of smear-negative pulmonary tuberculosis. Int J Tuberc Lung Dis. 1999;3(5):421-425. https:// pubmed.ncbi.nlm.nih.gov/10331732/

21. Bergmann JS, Woods GL. Clinical Evaluation of the BDProbeTec strand displacement amplification assay for rapid diagnosis of tuberculosis. $J$ Clin Micro. 1998;36(9):2766-2768. doi: 10.1128/JCM.36.9.27662768.1998

22. Spargo CA, Fraiser MS, Van Cleve M, et al. Detection of Mycobacterium tuberculosis DNA using thermophilic strand displacement amplification. Mol Cell Probes. 1996;10(4):247-256. doi: 10.1006/mcpr.1996.0034

23. Little MC, Andrews J, Moore R, et al. Strand displacement amplification and homogeneous real-time detection incorporated in a secondgeneration DNA probe system, BDProbeTec ET. Clin Chem. 1999;45(6 Pt 1):777-784. doi: 10.1093/ clinchem/45.6.777

24. Salfinger, M. Molecular laboratory testing for tuberculosis: innovators, early adopters, or laggards? Clin Infect Dis. 2014;58(7):977-979. doi: 10.1093/cid/ ciu024

25. Migliori GB, Matteelli A, Cirillo D, Pai M. Diagnosis of multidrug-resistant tuberculosis and extensively drug-resistant tuberculosis: current standards and challenges. Can J Infect Dis Med Microbiol. 2008;19(2):169-172. doi: 10.1155/2008/857901

26. World Health Organization (WHO). Molecular line probe assays for rapid screening of patients at risk of multi-drug resistant tuberculosis (MDR-TB). Expert group report. In: World Health Organization. Geneva, Switzerland. 2008. https://www.who.int/tb/features_ archive/policy_statement.pdf

27. De Beenhouwer H, Lhiang Z, Jannes $G$, et al. Rapid detection of rifampicin resistance in sputum and biopsy specimens from tuberculosis patients by PCR and line probe assay. Tuber Lung Dis. 1995;76(5):425430. doi: 10.1016/0962-8479(95)90009-8

28. Hillemann D, Weizenegger M, Kubica T, Richter E, Niemann S. Use of the genotype MTBDR assay for rapid detection of rifampin and isoniazid resistance in Mycobacterium tuberculosis complex isolates. J Clin Microbiol. 2005;43(8):3699-3703. doi: 10.1128/ JCM.43.8.3699-3703.2005 
29. The use of molecular line probe assay for the detection of resistance to isoniazid and rifampicin. Accessed June 1, 2017. https://www.who.int/publications/i/ item/9789241511261

30. Somoskovi A, Dormandy J, Rivenburg J, Pedrosa M, McBride M, Salfinger MM. Direct comparison of the GenoType MTBC and genomic deletion assays in terms of ability to distinguish between members of the Mycobacterium tuberculosis complex in clinical isolates and in clinical specimens. J Clin Microbiol. 2008;46(5):1854-1857. doi: 10.1128/JCM.00105-07

31. Tomasicchio $M$, Theron $G$, Pietersen $E$, et al. The diagnostic accuracy of the MTBDRplus and MTBDRsI assays for drug-resistant TB detection when performed on sputum and culture isolates. Sci Rep. 2016;6:17850. doi: $10.1038 /$ srep 17850

32. Meaza A, Kebede A, Yaregal Z, et al. Evaluation of genotype MTBDRplus VER 2.0 line probe assay for the detection of MDR-TB in smear positive and negative sputum samples. BMC Infect Dis. 2017;17(1):280. doi: 10.1186/s12879-017-2389-6

33. GenoType MTBDRsI | Detection of resistance of MTBC complex. Accessed November 24, 2021. https:// www.hain-lifescience.de/en/products/microbiology/ mycobacteria/tuberculosis/genotype-mtbdrsl.html

34. Hain Life Science. GenoType MTBDRsIVER 2.0 instructions for use. Document IFU-317A-01. HAIN LifeScience, Nehren, Germany. 2015. http://www. hain-lifescience.de/en/instructions-for-use.html.

35. World Health Organization (WHO). The use of molecular line probe assays for the detection of resistance to second-line anti-tuberculosis drugs-policy guidance. Geneva: WHO; 2016. https://apps.who.int/ iris/handle/10665/246131

36. Lawn SD, Nicol MP. Xpert ${ }^{\circledR}$ MTB/RIF assay: development, evaluation and implementation of a new rapid molecular diagnostic for tuberculosis and rifampicin resistance [published correction appears in Future Microbiol. 2012 Aug;7(8):1024]. Future Microbiol. 2011;6(9):1067-1082. doi:10.2217/fmb.11.84

37. Steingart KR, Schiller I, Horne DJ, Pai M, Boehme CC, Dendukuri N. Xpert MTB/RIF assay for pulmonary tuberculosis and rifampicin resistance in adults. Cochrane Database Syst Rev. 2014;21(1):1-16. doi: 10.1002/14651858.CD009593.pub3

38. World Health Organization (WHO). Automated realtime nucleic acid amplification technology for rapid and simultaneous detection of tuberculosis and rifampicin resistance: Xpert MTB/RIF system for the diagnosis of pulmonary and extrapulmonary TB in adults and children. WHO/HTM/TB/2013.14. Geneva, Switzerland: WHO; 2013. https://apps.who.int/iris/ handle/10665/112472

39. FDA permits marketing of first US test labeled for simultaneous detection of tuberculosis bacteria and resistance to the antibiotic rifampin - PubMed. https:// pubmed.ncbi.nlm.nih.gov/24195114/

40. Xpert MTB/RIF assay for the diagnosis of pulmonary and extrapulmonary TB in adults and children. Accessed December 15, 2015. https://www.who.int/ publications/i/item/9789241506335

41. Blakemore R, Story E, Helb D, et al. Evaluation of the analytical performance of the Xpert MTB/RIF assay. $J$ Clin Microbiol. 2010;48(7):2495-2501. doi: 10.1128/ JCM.00128-10

42. Iram S, Zeenat A, Hussain S, Yusuf NW, Aslam M. Rapid diagnosis of tuberculosis using Xpert MTB/RIF assay- report from a developing country. PakJ Med Sci. 2015;31(1):105-110. doi: 10.12669/pjms.311.6970

43. Lombardi G, Di Gregori V, Girometti N, Tadolini M, Bisognin F, Dal Monte P. Diagnosis of smear-negative tuberculosis is greatly improved by Xpert MTB/RIF. PLoS One. 2017;12(4):e0176186. doi: 10.1371/journal. pone. 0176186

44. Boehme CC, Nicol MP, Nabeta P, et al. Feasibility, diagnostic accuracy, and effectiveness of decentralised use of the Xpert MTB/RIF test for diagnosis of tuberculosis and multidrug resistance: a multicentre implementation study. Lancet. 2011;377(9776):14951505. doi: 10.1016/S0140-6736(11)60438-8

45. Xpert MTB/RIF implementation manual: technical and operational 'how-to'; practical considerations. Accessed October 10, 2021. https://apps.who.int/iris/ handle/10665/112469?search-result=true\&query $=X p$ ert+MTB\%2FRIF+implementation+manual\&scope $=\& r$ $\mathrm{pp}=10 \&$ sort_by=score \&order=desc

46. Centers for Disease Control and Prevention. Availability of an assay for detecting Mycobacterium tuberculosis, including rifampin-resistant strains, and considerations for its use-United States, 2013. MMWR Morb. Mortal Wkly. Rep. 2013;62(41):821-824. https://www.cdc. gov/mmwr/preview/mmwrhtml/mm6241a1.htm

47. Zetola NM, Shin SS, Tumedi KA, et al. Mixed Mycobacterium tuberculosis complex infections and false negative results for Gene Xpert MTB/RIF rifampinresistance are associated with poor clinical outcomes. J Clin Microbiol. 2014;52(7):2422-2429. doi: 10.1128/ JCM.02489-13

48. Rufai SB, Kumar P, Singh A, Prajapati S, Balooni V, Singh $S$. Comparison of Xpert MTB/RIF with line probe assay for detection of rifampicin monoresistant Mycobacterium tuberculosis. J Clin Microbiol. 2014;52(6):1846-1852. doi: 10.1128/JCM.03005-13

49. Notomi T, Okayama $\mathrm{H}$, Masubuchi $\mathrm{H}$, et al. Loopmediated isothermal amplification of DNA. Nucleic Acids Res. 2000;28(12):E63. doi: 10.1093/nar/28.12. e63

50. Parida M, Sannarangaiah S, Dash PK, Rao PVL, Morita K. Loop mediated isothermal Amplifcation (LAMP): a new generation of innovative gene amplifcation technique; perspectives in clinical diagnosis of infectious diseases. Rev Med Virol. 2008;18(6):407-421. doi: 10.1002/ rmv.593

51. Bojang AL, Mendy FS, Tientcheu LD, et al. Comparison of TB-LAMP, GeneXpert MTB/RIF and culture for diagnosis of pulmonary tuberculosis in the Gambia. I Infect. 2016;72(3):332-337. doi: 10.1016/j. jinf.2015.11.011

52. Ghosh PK, Chakraborty B, Maiti PK, Ray R. Comparative evaluation of loop-mediated isothermal amplification and conventional methods to diagnose extrapulmonary tuberculosis. Ann Trop Med Public Health. 2017;10(1):160-164. doi: 10.4103/17556783.205563 
53. World Health Organization (WHO). The use of Loopmediated Isothermal Amplification (TB-LAMP) for the diagnosis of pulmonary tuberculosis: Policy guidance. 2016. https://pubmed.ncbi.nlm.nih.gov/27606385/

54. Nagai K, Horita N, Yamamoto M, et al. Diagnostic test accuracy of loop-mediated isothermal amplification assay for Mycobacterium tuberculosis: systematic review and meta-analysis. Sci Rep. 2016;6:39090. doi: 10.1038/srep39090

55. COBAS TaqMan MTB Test - Free Download PDF. Accessed November 20, 2021. https://silo.tips/ download/cobas-taqman-mtb-test

56. Lee MR, Chung KP, Wang HC, Let al. Evaluation of the Cobas TaqMan MTB real-time PCR assay for direct detection of Mycobacterium tuberculosis in respiratory specimens. J Med Microbiol. 2013;62(8):1160-1164. doi: 10.1099/jmm.0.052043-0

57. Jonssona B, Lonnemark E, Ridell M. Evaluation of the Cobas TaqMan MTB test for detection of Mycobacterium tuberculosis complex. Infect Dis (Lond). 2015;47(4):231236. doi: $10.3109 / 00365548.2014 .987162$

58. Huh HJ, Koh WJ, Song DJ, Ki CS, Lee NY. Evaluation of the Cobas TaqMan MTB test for the detection of Mycobacterium tuberculosis complex according to acid-fast-bacillus smear grades in respiratory specimens. J Clin Microbiol. 2015;53(2):696-698. doi: 10.1128/JCM.02630-14

59. Park KS, Kim JY, Lee JW, et al. Comparison of the Xpert MTB/RIF and COBAS TaqMan MTB assays for detection of Mycobacterium tuberculosis in respiratory specimens. J Clin Microbiol. 2013;51(10):3225-3227. doi: 10.1128/JCM.01335-13

60. Horita N, Yamamoto M, Sato T, et al. Sensitivity and specificity of Cobas TaqMan MTB real-time polymerase chain reaction for culture-proven Mycobacterium tuberculosis: meta-analysis of 26999 specimens from 17 Studies. Sci Rep. 2015;5:18113. Published 2015 Dec 9. doi:10.1038/srep18113

61. Dorman SE, Schumacher SG, Alland D, et al. Xpert MTB/ RIF Ultra for detection of Mycobacterium tuberculosis and rifampicin resistance: a prospective multicentre diagnostic accuracy study. Lancet Infect Dis. 2018;18(1):76-84. doi: 10.1016/S14733099(17)30691-6

62. Chakravorty S, Simmons AM, Rowneki M, et al. The new Xpert MTB/RIF ultra: improving detection of Mycobacterium tuberculosis and resistance to rifampin in an assay suitable for point-of-care testing. MBio. 2017;8(4):e00812-e0817. doi: 10.1128/mBio.0081217

63. World Health Organization (WHO). Next-generation Xpert ${ }^{\circledR}$ MTB/RIF Ultra assay recommended by WHO. 2017. http://who.int/tb/features_archive/Xpert-Ultra/ en/

64. Garcia-Basteiro AL, Di Nardod A, Saavedraa B, et al. Point of care diagnostics for tuberculosis. Pulmonology. 2018;24(2):73-85. doi: 10.1016/j.rppnen.2017.12.002

65. Denkinger C. The TB diagnose pipeline. Cape Town, South Africa: $46^{\text {th }}$ Union World Conference on Lung Health; 2015. https://theunion.org/our-work/ conferences/history-of-the-union-world-conferenceon-lung-health
66. MacLean E, Huddart S, Pai M. Molecular diagnosis of tuberculosis: we need solutions that span the healthcare value chain. Expert Rev Mol Diagn. 2017;17(1):5-7. doi: 10.1080/14737159.2017.1265889

67. (PDF) Pre-validation of the BD MAX MDR-TB* assay for the rapid detection of MTBC DNA and mutations associated with rifampin and isoniazid resistance Introduction Results. Accessed September 17, 2018. https://www.researchgate. net/publication/324804012_Pre-validation_of_the_ BD_MAX_MDR-TB_assay_for_the_rapid_detection of_MTBC_DNA_and_mutations_associated_with_ rifampin_and_isoniazid_resistance_Introduction _ Results

68. Mitarai S, Okumura M, Toyota $E$, et al. Evaluation of a simple loop-mediated isothermal amplification test kit for the diagnosis of tuberculosis. Int J Tuberc Lung Dis. 2011;15(9):1211-1217. doi: 10.5588/ijtld.10.0629

69. Agarwal A, Gaurav SG, Jasuj ND. Analysis of diagnostic methods and their sensitivity test for Mycobacterium tuberculosis. SGVU Int J Env Sci Technol. 2016;2(1):1727. https://www.gyanvihar.org/researchjournals/ ApurvaVolume\%202,\%20lssue\%201,\%202016.pdf

70. KouzakiY, Mikita K, Maeda T, etal. PURE-LAMP procedure for the diagnosis of Extrapulmonary tuberculosis: a case series. Intern Med. 2015;54(11):1447-1450. doi: 10.2169/internalmedicine.54.4615

71. French DJ, Archard CL, Brown T, McDowell DG. HyBeacon probes: a new tool for DNA sequence detection and allele discrimination. Mol Cell Probes. 2001;15(6):363-374. doi: 10.1006/mcpr.2001.0384

72. Hofmann-Thiel S, Hoffmann H. Evaluation of Fluorotype MTB for detection of Mycobacterium tuberculosis complex DNA in clinical specimens from a low-incidence country. BMC Infect Dis. 2014;14:59. doi: 10.1186/1471-2334-14-59

73. FluoroType ${ }^{\circledR}$ MTB | Detection of M. tuberculosis from patient specimens. Accessed December 25, 2021. https://www.hain-lifescience.de/en/products/ microbiology/mycobacteria/tuberculosis/fluorotypemtb.html

74. Nikam C, Kazi M, Nair C, et al. Evaluation of the Indian TrueNAT micro RT-PCR device with GeneXpert for case detection of pulmonary tuberculosis. Int J Mycobacteriol. 2014;3(3):205-210. doi: 10.1016/j. ijmyco.2014.04.003

75. Tripathy $S$, et al. Final report on operational feasibility and performance of TrueNatMTB/Rif assays in field settings under the Revised National Tuberculosis Control Program. New Delhi: Indian Council of Medical Research. 2016. https:// www.molbiodiagnostics.com/uploads/product evaluation/2_productevaluation_220190405.134141. pdf

76. Nikam C, Jagannath M, Narayanan MM, et al. Rapid Diagnosis of Mycobacterium tuberculosis with Truenat MTB: a near-care approach. PLOS ONE. 2013;8(1):e51121. doi: 10.1371/journal.pone.0051121

77. Papaventsis D, Casali N, Kontsevaya I, Drobniewski F, Cirillo DM, Nikolayevskyy V. Whole genome sequencing of Mycobacterium tuberculosis for detection of drug resistance: a systematic review. Clin Microbiol Infect. 
2017;23(2):61-68. doi: 10.1016/j.cmi.2016.09.008

78. Kwong JC, McCallum N, Sintchenko V, Howden BP. Whole genome sequencing in clinical and public health microbiology. Pathology. 2015;47(3):199-210. doi: 10.1097/PAT.0000000000000235

79. Walker TM, Ip CL, Harrell $\mathrm{RH}$, et al. Whole-genome sequencing to delineate Mycobacterium tuberculosis outbreaks: a retrospective observational study. Lancet Infect Dis. 2013;13(2):137-146. doi: 10.1016/S14733099(12)70277-3

80. Koser CU, Ellington MJ, Peacock SJ. Whole-genome sequencing to control antimicrobial resistance. Trends Genet. 2014;30(9):401-407. doi: 10.1016/j. tig.2014.07.003

81. Bradley SP, Reed SL, Catanzaro A. Clinical efficacy of the amplified Mycobacterium tuberculosis direct test for the diagnosis of pulmonary tuberculosis. Am J Respir Crit Care Med. 1996;153(5):1606-1610. doi: 10.1164/ ajrccm.153.5.8630609

82. Bloemberg GV, Voit A, Ritter C, Deggim V, Bottger EC. Evaluation of Cobas TaqMan MTB for Direct Detection of the Mycobacterium tuberculosis Complex in Comparison with Cobas Amplicor MTB. J Clin Microbiol. 2013;51(7):2112-2117. doi: 10.1128/JCM.00142-13

83. Rohner P, Jahn EI, Ninet B, et al. Rapid Diagnosis of Pulmonary Tuberculosis with the LCX Mycobacterium tuberculosis Assay and Comparison with Conventional Diagnostic Techniques. J Clin
Microbiol. 1998;36(10):3046-3047. doi: 10.1128/ JCM.36.10.3046-3047.1998

84. McHugh TD, Pope $C F$, Ling $C L$, et al. Prospective evaluation of BDProbeTec strand displacement amplification (SDA) system for diagnosis of tuberculosis in non-respiratory and respiratory samples. J Med Microbiol. 2004;53(12):1215-1219. doi: 10.1099/ jmm.0.45780-0

85. Mekkaoui L, Hallin M, Mouchet F, et al. Performance of Xpert MTB/RIF Ultra for diagnosis of pulmonary and extra-pulmonary tuberculosis, one year of use in a multi-centric hospital laboratory in Brussels, Belgium. PLOSONE. 2021;16(4):e0249734. doi: 10.1371/journal. pone. 0249734

86. Shah M, Paradis S, Betz J, et al. Multicenter Study of the Accuracy of the BD MAX Multidrug-resistant Tuberculosis Assay for Detection of Mycobacterium tuberculosis Complex and Mutations Associated With Resistance to Rifampin and Isoniazid. Clin Infect Dis. 2020;71(5):1161-1167. doi: 10.1093/cid/ciz932

87. Chen X, He G, Wang S, Lin S, Chen J, Zhang W. Evaluation of Whole-Genome Sequence Method to Diagnose Resistance of 13 Anti-tuberculosis Drugs and Characterize Resistance Genes in Clinical Multi-Drug Resistance Mycobacterium tuberculosis Isolates From China. Front Microbiol. 2019;10:1741. doi: 10.3389/ fmicb.2019.01741 